\title{
Interaction Between Diaporthe rhusicola and Neofusicoccum mediterraneum Causing Branch Dieback and Fruit Blight of English Walnut in California, and the Effect of Pruning Wounds on the Infection
}

\author{
Carlos Agustí-Brisach, ${ }^{1,2}$ Juan Moral, ${ }^{1,2}$ Dan Felts, ${ }^{1}$ Antonio Trapero, ${ }^{2}$ and Themis J. Michailides, ${ }^{1 \dagger}$ \\ ${ }^{1}$ Kearney Agricultural Research and Extension Center, University of California, Davis, Parlier, CA 93648, U.S.A.; and \\ ${ }^{2}$ Departamento de Agronomía, Escuela Técnica Superior de Ingenieros Agrónomos y de Montes, Universidad de Córdoba, \\ Campus de Rabanales, 14071 Córdoba, Spain
}

\begin{abstract}
Botryosphaeriaceae and Diaporthaceae species are the causal agents of branch dieback of English walnut in California. In this study, the effects of the interaction between Neofusicoccum mediterraneum and Diaporthe rhusicola were evaluated in vitro by using mycelial plugs or spore suspensions and in vivo by inoculating shoots and epicarps (hulls) of walnut. Single inoculations of each species and different coinfection treatments were performed under laboratory or field conditions. The influence of shoot age and susceptibility of bark or pith tissues to $N$. mediterraneum and $D$. rhusicola infection after pruning was also evaluated. In in vitro experiments,

and hulls was observed compared with the other two interaction treatments. One- to 2-year-old shoots were more susceptible to infection and colonization by $N$. mediterraneum than 3- to 4-year-old shoots. In young shoots, inoculation in the pith tissue resulted in longer lesions than those observed on shoots inoculated in the bark. No significant differences were observed between the development of internal or external necrosis and the age of the shoots, or the susceptibility of bark and pith to D. rhusicola infection. This information is essential to better understanding the complex situation of this walnut disease toward developing control management strategies.
\end{abstract} spore germination of $D$. rhusicola was significantly $(P<0.0001)$ reduced in the presence of $N$. mediterraneum spores. When D. rhusicola was inoculated at 4 days before $N$. mediterraneum, a delay in lesion development in shoots
Keywords: canker disease, Juglans regia, pathogen interactions, Phomopis rhusicola, tree nuts
English walnut (Juglans regia L.) represents one of the major nut crops produced in the United States. California leads the production of English walnut in the United States, with $99 \%$ of total U.S. production and $38 \%$ of global production and more than 30 cultivars planted (Chen et al. 2014a; Olson and Buchner 2002). Approximately 95\% of walnuts in California are grown in the Central and Sacramento valleys, comprising the area between Tulare County (to the south) and Tehama County (to the north) (Olson and Buchner 2002). In 2016, 686,000 tons of walnuts were produced on 127,475 hectares in California, with an estimated value of $\$ 1,241.66$ million (California Department of Food and Agriculture 2017).

During late spring and summer of 2003, shoot blights and cankers in large dead branches were observed for the first time on English walnut in orchards located in Yuba, Sutter, and Stanislaus counties in California. Since then, a large number of blighted shoots of Chandler, Tulare, and other walnut cultivars have consistently been found in orchards from the main walnut growing counties in the San

Current address for C. Agustí-Brisach: Departamento de Agronomía, Escuela Técnica Superior de Ingenieros Agrónomos y de Montes, Universidad de Córdoba, Campus de Rabanales, 14071 Córdoba, Spain.

${ }^{\dagger}$ Corresponding author: T. J. Michailides; tjmichailides@ucanr.edu

Funding: This research was funded by a California Walnut Board production research grant (CWB Project 2016-2018-Michailides). C. Agustí-Brisach holds a Juan de la Cierva-Incorporación fellowship from the Spanish Ministry for the Economy, Industry and Competitiveness, and his stay at the Kearney Agricultural Research and Extension Center was supported partially by a California Walnut Board production research grant to T. Michailides. J. Moral holds a Marie Skłodowska-Curie fellowship launched by the European Union's Horizon 2020 (contract number 658579).

The author(s) declare no conflict of interest.

Accepted for publication 24 October 2018.

@ 2019 The American Phytopathological Society
Joaquín and Sacramento valleys, such as Sutter, Yuba, Stanislaus, Tulare, Kings, Glenn, and Yolo counties (Michailides and Hasey 2010). Typical dieback symptoms include leaf blight in association with shoot blight, wood canker formation, and blight affecting the spur, twig, fruit, and bud (Chen et al. 2014a; Trouillas et al. 2010). The initial conspicuous symptom of branch infection is often wilting and flagging of leaves on branches distal to the canker. The stems generally have discolored areas on the bark. Cutting away the bark reveals cortical and cambial tissues that are discolored from brown to black. If the infection is old enough, peeling the bark reveals well-developed embedded black pycnidia (fruiting bodies) of the pathogens. When temperatures increase in late spring and summer, the symptoms progress quickly and the affected branches consequently die (Michailides and Hasey 2010).

Botryosphaeriaceae and Diaporthaceae species have been described as the main causal agents of branch dieback, and they are now widely distributed in English walnut orchards in all of the Californian counties where walnuts are grown (Chen et al. 2014a). In fact, $\sim 70 \%$ of the diagnosed samples were infected by Botryosphaeriaceae, $10 \%$ were infected by Diaporthe fungi, and $20 \%$ showed coinfections by the two groups of fungi (T. J. Michailides, unpublished data). It is well known that fungal species belonging to the Botryosphaeriaceae family cause twig and branch dieback symptoms on a broad range of woody plants worldwide (Slippers and Wingfield 2007). In California, many important fruit and nut trees, such as almond (Prunus dulcis L.; Inderbitzin et al. 2010), avocado (Persea Americana Mill.; McDonald and Eskalen 2011), grapevine (Vitis vinifera L.; Úrbez-Torres and Gubler 2009; Úrbez-Torres et al. 2006), olive (Olea europaea ssp. europaea L.; Moral et al. 2010; ÚrbezTorres et al. 2013), and pistachio (Pistacia vera L.; Chen et al. 2014b; Ma and Michailides 2002), are affected by Botryosphaeriaceae fungi. In California, studies conducted by Chen et al. (2014a) in English walnut orchards showed 10 species of Botryosphaeriaceae associated with this crop: Botryosphaeria dothidea, Diplodia mutila, Diplodia seriata, Dothiorella iberica, Lasiodiplodia citricola, Neofusicoccum mediterraneum, Neofusicoccum nonquaesitum, Neofusicoccum parvum, Neofusicoccum vitifusiforme, and Neoscytalidium 
dimidiatum. Among them, $N$. mediterraneum is considered the most frequent species recovered from affected tissues of walnut, and it is widely distributed in commercial orchards. Pathogenicity tests using walnut shoots revealed that all of these species were pathogenic, with $L$. citricola being the most aggressive species, followed by $N$. parvum (Chen et al. 2014a). In addition, B. dothidea and L. pseudotheobromae have been associated with branch dieback of English walnut in China (Li et al. 2015). Diaporthe species have also been reported to cause branch dieback and cankers, fruit rot, leaf spot, or shoot blight in other nut crops such as almond or pistachio (Gramaje et al. 2012; Teviotdale et al. 2002). Among species belonging to the Diaporthe genus, D. neotheicola and D. rhusicola have been associated with branch dieback of English walnut in California. However, pathogenicity tests revealed that they are weakly pathogenic to English walnut shoots (Chen et al. 2014a).

Botryosphaeriaceae and Diaporthe species develop and survive as saprophytic microorganisms on dead plant tissues such as the bark of trees. When the affected tissues die, the pathogens develop fruiting bodies on dead tissues that can produce viable spores over a period of years. Thus, they provide the spore sources that cause the disease on English walnut trees. These spores are able to enter the tree through natural wounds (e.g., the fruit abscission point, leaf scars, tissues damaged by walnut scale infestation, etc.) or artificial wounds (e.g., pruning wounds) caused on shoots and branches. Once the spores get inside the bark of healthy trees, they remain dormant until conditions are right for them to germinate and cause disease (Michailides and Hasey 2010; J. Moral et al., unpublished data).

These previous studies indicate that Botryosphaeriaceae species are the main causal agents of branch dieback and suggest that Diaporthaceae species could act as saprophytes or incite disease development when coinfections with Botryosphaeriaceae species occur. However, the effect of the interaction between Botryosphaeriaceae and Diaporthaceae species is still unknown. Moreover, the effect of pruning wounds on pathogen infections, the susceptibility of the wounded tissues after pruning (e.g., bark or pith), and the influence of the age of the shoots of English walnut to be infected by Botryosphaeriaceae and Diaporthaceae species have not yet been studied. Thus, the objectives of this study were as follows: (i) to evaluate the effect of temperature on the mycelial growth in vitro of $D$. rhusicola and $N$. mediterraneum isolates from walnut; (ii) to elucidate the interaction between $D$. rhusicola and $N$. mediterraneum in the coinfection of shoots of walnut inoculated with mycelial plugs and conidial suspensions; (iii) to evaluate the interaction between $D$. rhusicola and $N$. mediterraneum in the infection of detached walnut hulls inoculated with conidial suspensions; and (iv) to compare the influence of the age and the susceptibility of different pruned tissues (bark or pith) of the shoots of English walnut to D. rhusicola and $N$. mediterraneum infection after pruning.

\section{Materials and Methods}

Fungal isolates. Two isolates each of $D$. rhusicola (isolates $6 \mathrm{I} 31$ and 6I44) and N. mediterraneum (isolates 5C87 and 6I45), all isolated from diseased walnuts in California and stored in the Michailides fungal collection at the Kearney Agricultural and Extension Research Center at University of California, Davis (KARE), in Parlier, California, were used in the various experiments in this study. They were grown on potato dextrose agar (PDA) (Microtech Scientific, Orange, CA) at $25^{\circ} \mathrm{C}$ for 7 days in darkness and also on pistachio leaf agar (10 $\mathrm{g}$ of PDA, $10 \mathrm{~g}$ of bacteriological agar from Microtech Scientific, 1 liter of distilled water, and one twicesterilized pistachio leaflet per Petri dish) at $25^{\circ} \mathrm{C}$ for 3 weeks under continuous fluorescent light to induce pycnidia production.

Effect of temperature on mycelial growth. A mycelial plug ( $5 \mathrm{~mm}$ in diameter) obtained from the margins of 10-day-old actively growing colonies of the two representative isolates of $D$. rhusicola and $N$. mediterraneum growing on PDA was placed in the center of each Petri dish filled with PDA. Petri dishes were incubated at $5,10,15,20,25,30$, and $35^{\circ} \mathrm{C}$ in darkness. The diameter of the two perpendicular axes was measured daily for 5 and 9 days for $N$. mediterraneum and D. rhusicola isolates, respectively. Mean data were converted to the radial growth rate (in millimeters per day). Five Petri dishes per isolate and temperature were used as replicates, and the experiment was repeated twice.

For each isolate, a nonlinear adjustment of the data was applied using the generalized analytics beta model (Hau and Kranz 1990) to evaluate variation in the mycelial growth rate over temperature (LópezMoral et al. 2017). For each isolate, temperature mean growth rates were adjusted to a regression curve to estimate the optimum growth temperature $\left({ }^{\circ} \mathrm{C}\right)$ for radial growth, maximum daily radial growth (millimeters per day), and the relative area under the mycelial growth curve (RAUMGC; \%). Analysis of variance (ANOVA) was performed on the three parameters and isolate means were compared according to Fisher's protected least significant difference (LSD) test at $P=$ 0.05 (Steel and Torrie 1985). Data from this experiment were analyzed using Statistix 10 (Analytical Software 2013).

Interaction between $D$. rhusicola and $N$. mediterraneum. In vitro experiments. A conidial suspension $\left(10^{5}\right.$ conidia $\left.\mathrm{ml}^{-1}\right)$ was obtained by scraping the mycelial and pycnidia masses of $D$. rhusicola isolate $6 \mathrm{I} 31$ and $N$. mediterraneum isolate 5C87 developed on the surface of leaf agar after 3 weeks under continuous fluorescent light. Drops $(20 \mu \mathrm{l})$ of the conidial suspension of each isolate were placed in the center of a microscope coverslip $(20 \times 20 \mathrm{~mm})$. For the interaction treatment, a drop $(10 \mu \mathrm{l})$ of each isolate conidial suspension was placed in the same microscope coverslip and homogenized using a glass rod. Three coverslips per treatment were placed inside Petri dishes containing 2\% water agar (Microtech Scientific) and were incubated at $25^{\circ} \mathrm{C}$ (Moral et al. 2010). One coverslip from each treatment was removed after 24 and $48 \mathrm{~h}$ of incubation. Spore germination was stopped by staining with a drop of $0.01 \%$ fuchsine acid in lactoglycerol (1:2:1 lactic acid/glycerol/water) and the percentage of spore germination was determined by observing 100 conidia selected at random on each coverslip. A spore was considered germinated if the germ tube was at least one-half the longitudinal axis of the spore. For each treatment, the mean germination was obtained at the maximum incubation time of $48 \mathrm{~h}$ (Moral et al. 2010). The experiment was conducted twice. Data from both experiments were averaged because there were no significant differences between them ( $P=0.7160)$ according to the LSD test at $P=0.05$. Germination data were subjected to a factorial ANOVA in order to determine the differences among fungal species, interaction treatment, incubation time, and their interactions. Treatment means of germination (in percentages) were compared separately for each fungal species and incubation time according to the chi-square test at $P=0.05$.

Interaction effect on walnut infection: Inoculation treatments. A total of 16 different treatments were conducted using the four representative isolates selected in this study: (i) single inoculations of each isolate (four treatments in total); (ii) coinoculations with both species at different times, with the second fungal species being inoculated 4 days after the first one (the two species $D$. rhusicola and $N$. mediterraneum, the two representative isolates of each one, and the two inoculation times combined between them; eight treatments in total); and (iii) inoculations with both species at the same time (the two species and the two representative isolates of each one were combined between them; four treatments in total). In addition, the pathogens were inoculated with mycelial plugs or with conidial suspensions in order to compare the effect of the inoculation mode. Noninoculated treatment was conducted as the control. The experiments were conducted on detached shoots under controlled laboratory conditions as well as on attached shoots in natural field conditions, as described below.

Inoculation on detached shoots. In April 2017, 1- to 2-year-old detached shoots of walnut cultivar Vina were collected from the KARE experimental orchards located in Parlier, California. Shoot segments $(\approx 25 \mathrm{~cm}$ length) were sealed at both ends with Parafilm (Bemis, Neenah, WI) to reduce desiccation and were maintained at $4{ }^{\circ} \mathrm{C}$ until they were processed. The bark surface was sterilized by dipping in a $70 \%$ ethanol solution for $1 \mathrm{~min}$ and was air dried on a laboratory bench for $30 \mathrm{~min}$. The outer bark was removed in the center of the branch segment using a 5-mm-diameter cork borer. In the case of interaction treatments (coinoculations), two parallel holes were made next to each other. Shoot segments were placed in humid chambers (plastic 
containers, $30 \times 23 \times 10 \mathrm{~cm}$ ) and inoculated with either a 5-mmdiameter mycelium plug obtained from the active growing margin of the fungal colony or by deposition of a $10-\mu l$ drop of a conidial suspension adjusted with a hemacytometer to $5 \times 10^{5}$ conidia ml $^{-1}$ on the wound. Then the inoculated area was wrapped with Parafilm, and the inoculated shoots were incubated at $27 \pm 2{ }^{\circ} \mathrm{C}$ and $100 \%$ relative humidity for 1 month. There were three replicated humid chambers per treatment and inoculation mode, with five shoot segments per humid chamber and 15 shoot segments per treatment and inoculation mode. Fifteen shoot segments treated with a 5-mm-diameter PDA plug or a $10-\mu l$ drop of sterile distilled water (SDW) were included as the control. A completely randomized design was used for each inoculation mode, with treatment as the independent variable and humid chambers as replications. For each shoot segment, evaluation of the lesion length was recorded at 4 weeks after inoculation. Small wood fragments from the margin of the affected area of each inoculated branch were surface disinfested by dipping in a commercial bleach $(\mathrm{Cl}$ at $50 \mathrm{~g}$ per liter) solution at $10 \%$ (vol/vol) in sterile water for $2 \mathrm{~min}$, air dried on sterile filter paper for $15 \mathrm{~min}$, and plated on PDA acidified with lactic acid $(2.5 \mathrm{ml}$ of $25 \%$ [vol/vol] per liter of medium) to reisolate the pathogens. For each inoculation mode (mycelial plug and conidial suspension), ANOVA of the dependent variable (lesion length) was performed to determine the differences in virulence among fungal isolates and their interactions. Data were tested for normality and homogeneity of variances and were logarithmically transformed. Control treatments were excluded from the statistical analysis because no lesions were observed in noninoculated control shoots. The treatment means were compared according to Fisher's protected LSD test at $P=0.05$.

Inoculation under field conditions. One- to 2-year-old shoots were selected in a 12-year-old KARE Vina walnut experimental orchard. Prior to making the 5-mm hole in the branch, the area of the inoculation site was surface disinfected by spraying with $70 \%$ ethanol solution. Subsequently, inoculations were conducted by using mycelial plugs or conidial suspensions as described above. This experiment
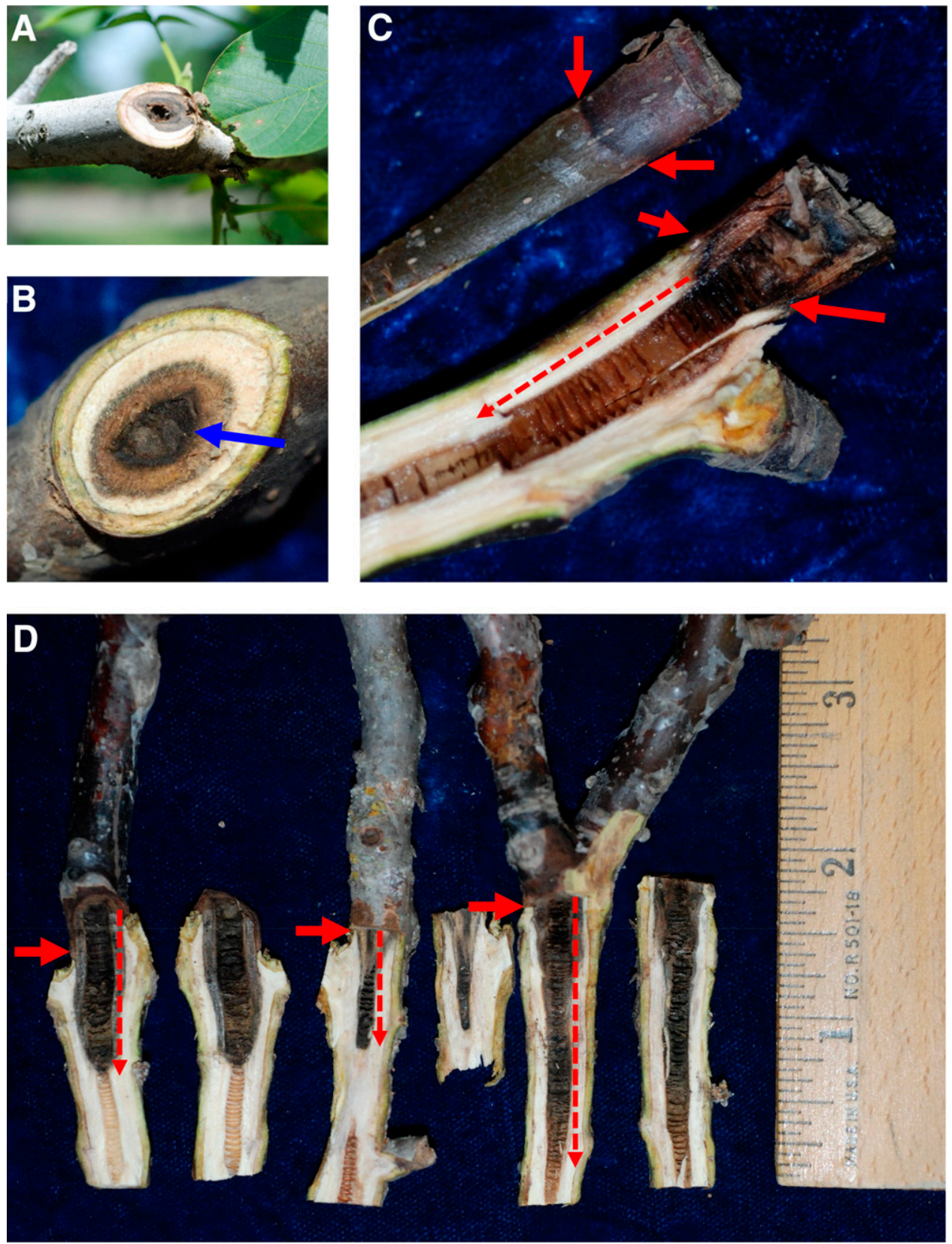

Fig. 1. A and B, Cankers in walnut shoots starting the infection and disease development from the pith area (blue arrow: wounded pith tissue). C and D, Longitudinal section of infected shoots showing the advance of Neofusicoccum mediterraneum infection in the pith area compared with the canker margin (red arrow: external lesion; discontinuous red arrow: internal lesion). 
began on 11 April 2017. There were 15 replicated trees per inoculation mode, with one inoculated branch per treatment in each tree and 15 inoculated shoots per treatment and inoculation mode (480 shoots in total). In addition, 15 shoots (one branch per replicated tree) treated with a 5-mm-diameter PDA plug or a 10- $\mu$ l drop of SDW were included as controls. For each inoculation mode, a completely randomized design was used, with treatment as the independent variable and trees as replications. For each branch, evaluation of the lesion length was recorded at 4 months after inoculation. Fungal reisolation was conducted from the margin and $2 \mathrm{~cm}$ beyond the margin as described above. Because heterogeneity of variances was observed, the Kruskal-Wallis one-way nonparametric test was performed for each inoculation mode (mycelial plug and conidial suspension) to determine the differences in virulence among fungal isolates and their interactions. In this case, treatment means were compared according to Dunn's test at $P=0.05$.

Inoculation of walnut fruit. In June 2017, green fruits of Vina walnut were collected from the KARE experimental orchard. The fruits were surface disinfested by dipping in a commercial bleach solution at $10 \%(\mathrm{vol} / \mathrm{vol})$ in sterile water for $2 \mathrm{~min}$, and they were air dried on sterile filter paper for $30 \mathrm{~min}$. Disinfested walnut fruits were placed in humid chambers and wounded by making a hole $5 \mathrm{~mm}$ in diameter and $4 \mathrm{~mm}$ deep on the center of the hull of each fruit using a cork borer. Subsequently, inoculations were conducted by placing a $10-\mu 1$ drop of a conidial suspension adjusted with a hemacytometer to $5 \times 10^{5}$ conidia $\mathrm{ml}^{-1}$ inside each hole. There were three replicated humid chambers per treatment, with 10 fruits per humid chamber and 30 fruits per treatment (480 fruits in total). In addition, 30 fruits treated with a $10-\mu 1$ drop of SDW were included as the control. A completely randomized design was used, with treatment as the independent variable and humid chambers as replications. Disease severity was evaluated in inoculated fruits until most of the hull surface (90 to 100\%) of the fruits was affected. Four evaluations were made in total. The major and minor diameters of each lesion were recorded and averaged for each evaluation, and the percentage of the affected hull surface of each fruit in each treatment and evaluation was calculated. The relative area under the disease progress curve (RAUDPC) was obtained by trapezoidal integration (Campbell and Madden 1990). RAUDPC data were subjected to a factorial ANOVA and the treatment means were compared according to Fisher's protected LSD test at $P=0.05$.

Effect of age and tissue of the pruned shoots on pathogen infection. In May 2017, shoots from two age categories (1 to 2 years and 3 to 4 years) of Chandler walnut were collected from the KARE experimental orchards. Shoot segments (»15 cm length) were sealed at both ends with Parafilm to reduce desiccation and were maintained at $4{ }^{\circ} \mathrm{C}$ until they were processed. The bark surface was sterilized as described above. Prior to inoculation, one of the ends of the shoot was cut with sterile pruning scissors, and shoot segments were placed in humid chambers. Inoculations were done with a $10-\mu l$ drop of $5 \times 10^{5}$ conidia $\mathrm{ml}^{-1}$ conidial suspension of $D$. rhusicola isolate $6 \mathrm{I} 31$ or $N$. mediterraneum isolate $5 \mathrm{C} 87$, which was deposited on the wounded bark or on the wounded pith (see the detail of wounded pith tissue in Fig. 1, A and B), in order to evaluate the effect of the infection site on disease development. Then inoculated shoots were incubated in humid chambers for 3 weeks as described above. There were three replicated humid chambers per fungal isolate, age category, and inoculation site (bark or pith), with five shoots per humid chamber and 15 shoots per treatment combination (120 shoots in total). In addition, 15 shoots per age category and inoculation site were treated with SDW as the control (60 control shoots in total). A completely randomized design was used, with combination treatment as the independent variable and humid chambers as replications. For each branch, evaluation of the lesion length was recorded at 2 and 3 weeks after inoculation. Lesion lengths on the surface (external lesion) and on the pith (internal lesion) were recorded. Fungal reisolation was conducted as described above. ANOVA of the dependent variable (lesion length) was performed to determine the differences in virulence among fungal species, age categories, inoculation site, and their interactions. Data were tested for normality and homogeneity of variances. Control treatments were excluded from the statistical analysis because no lesions were observed in noninoculated control shoots. The treatment means were compared according to Fisher's protected LSD test at $P=0.05$.

\section{Results}

Effect of temperature on mycelial growth. The isolates of $N$. mediterraneum were able to grow on PDA at 5 to $35^{\circ} \mathrm{C}$, showing little mycelial growth development at both extreme temperatures tested. However, D. rhusicola isolates did not grow at $35^{\circ} \mathrm{C}$; both isolates showed very little or poor mycelial growth development at $5^{\circ} \mathrm{C}$, and D. rhusicola isolate $6 \mathrm{I} 44$ also at $30^{\circ} \mathrm{C}$. Optimum growth temperatures ranged from 22.3 to $30.4^{\circ} \mathrm{C}$, and the maximum growth rate ranged from 3.0 to $7.2 \mathrm{~mm} \mathrm{day}^{-1}$ for isolates D. rhusicola $6 \mathrm{I} 44$ and $N$. mediterraneum 6I45, respectively (Table 1). Isolates D. rhusicola $6 \mathrm{I} 44$ and $N$. mediterraneum $6 \mathrm{I} 45$ showed an optimum growth temperature that was significantly lower $\left(22.3^{\circ} \mathrm{C} ; P<0.0001\right)$ and higher $\left(30.4^{\circ} \mathrm{C} ; P<0.0001\right)$, respectively, than the rest of the isolates, which did not differ among themselves. Concerning the maximum growth rate, significant differences $(P<0.0001)$ between isolates were observed. The two strains of $N$. mediterraneum grew more rapidly than the two strains of $D$. rhusicola, and there were also significant differences between the two strains of D. rhusicola (Table 1). Finally, when the RAUMGC was calculated, a different pattern from that observed for the maximum growth rate was obtained. In this last case, the two strains of $N$. mediterraneum had a higher AUC than the two strains of $D$. rhusicola, although there were significant differences between the strains of $N$. mediterraneum but not between those of D. rhusicola. RAUMGC values ranged from 33.0 to $100 \%$ for isolates $D$. rhusicola $6 \mathrm{I} 31$ and $N$. mediterraneum $5 \mathrm{C} 87$, respectively (Table 1).

Interaction between $D$. rhusicola and $N$. mediterraneum. In vitro experiments. Because the interactions between variables were significantly different $(P<0.05)$, treatment means of germination (in percentages) were compared separately for each fungal species and incubation time according to the chi-square test at $P=0.05$. Overall, the presence of $D$. rhusicola conidia on the mixed suspension of spores did not affect the percentage of germination of $N$.

Table 1. Effect of temperature on mycelial growth of Diaporthe rhusicola (isolates $6 \mathrm{I} 31$ and 6I44) and Neofusicoccum mediterraneum (isolates 5C87 and 6I45) grown on potato dextrose agar at $0,5,10,15,20,25,30$, and $35^{\circ} \mathrm{C}$ in darkness for 9 and 5 days, respectivelyw

\begin{tabular}{|c|c|c|c|c|c|c|c|c|c|}
\hline \multirow[b]{2}{*}{ Species } & \multirow[b]{2}{*}{ Isolate } & \multicolumn{3}{|c|}{ Analytics beta model ${ }^{\mathrm{x}}$} & \multicolumn{3}{|c|}{ Temperature $\left({ }^{\circ} \mathrm{C}\right)^{\mathrm{y}}$} & \multirow[b]{2}{*}{$\mathbf{M G R}^{\mathbf{z}}$} & \multirow[b]{2}{*}{ RAUMGC (\%) } \\
\hline & & $R^{2}$ & $a$ & $b$ & Optimum & Minimum & $\overline{\text { Maximum }}$ & & \\
\hline \multirow[t]{2}{*}{ D. rhusicola } & $6 \mathrm{I} 31$ & 0.9847 & 5.07 & 0.86 & $26.6 \mathrm{~b}$ & 3.5 & 30.5 & $4.1 \mathrm{~b}$ & $33.0 \mathrm{c}$ \\
\hline & $6 \mathrm{I} 44$ & 0.9937 & 3.27 & 1.69 & $22.3 \mathrm{c}$ & 4.5 & 31.5 & $3.0 \mathrm{c}$ & $33.2 \mathrm{c}$ \\
\hline \multirow[t]{2}{*}{ N. mediterraneum } & $5 \mathrm{C} 87$ & 0.9914 & 2.94 & 1.10 & $25.8 \mathrm{~b}$ & 0.0 & 35.5 & $7.0 \mathrm{a}$ & $100 \mathrm{a}$ \\
\hline & $6 \mathrm{I} 45$ & 0.9870 & 1.59 & 0.21 & $30.4 \mathrm{a}$ & 4.5 & 35.0 & $7.2 \mathrm{a}$ & $87.5 \mathrm{~b}$ \\
\hline
\end{tabular}

\footnotetext{
${ }^{\text {w }}$ Means in a column followed by the same lowercase letter do not differ significantly according to Fisher's protected least significant difference (LSD) at $P=0.05$ (Steel and Torrie 1985). RAUMGC $=$ area under the curve of mycelial growth over time $(\%)$.

${ }^{x}$ In the analytics beta model, $R^{2}$ is the coefficient of determination and $a$ and $b$ are the coefficients of regression.

${ }^{y}$ For each isolate, temperature average growth rates were adjusted to a regression curve to estimate the optimum growth temperature.

${ }^{\mathrm{z}}$ Maximum growth rate measured in millimeters per day.
} 
mediterraneum conidia (chi-square test, $P>0.05$ ). Thereafter, the average spore germination of $N$. mediterraneum obtained from the two incubation times was the same for the control and the interaction treatments $(71.4 \%)$. Conversely, the percentage of spore germination of $D$. rhusicola was markedly $(P<0.0001)$ lower when $N$. mediterraneum conidia were also present $(85.1 \%$ versus $21.1 \%$, respectively) (Table 2).

Interaction effect on walnut infection: Inoculation on detached shoots. Because the experiments were homogenous and there were no significant differences between the isolates of the same fungal species $(P=0.4501$ and $P=0.1419$ for $N$. mediterraneum and $D$. rhusicola isolates, respectively), the treatments were grouped per fungal species. However, experiments inoculated with mycelial plugs or conidial suspensions were analyzed separately, as there were significant $(P<0.0001)$ differences between the two inoculation methods. Regarding detached shoots inoculated with mycelial plugs, significant differences among treatments $(P<0.0001)$ were found. Inoculations conducted using only $N$. mediterraneum, and the interaction of $N$. mediterraneum and D. rhusicola when both species were inoculated at the same time or when $N$. mediterraneum was inoculated 4 days before $D$. rhusicola, showed the highest disease severity (ranging from 12.4 to $12.8 \mathrm{~cm}$ at 4 weeks after inoculation). Shoots only inoculated with $D$. rhusicola showed the lowest severity, with a necrosis length of $3.8 \mathrm{~cm}$. When $D$. rhusicola was inoculated at 4 days before $N$. mediterraneum, the effect of the interaction induced a major necrosis development (necrosis length of $9.8 \mathrm{~cm}$ at 4 weeks after inoculation) than that observed when $D$. rhusicola was inoculated alone. Nevertheless, the necrosis length in this interaction treatment was less than the length observed for the other two interaction treatments in combination with $N$. mediterraneum, suggesting a possible delay in lesion development probably owing to the fact that $N$. mediterraneum was inoculated 4 days after D. rhusicola (Fig. 2A). A similar pattern was observed in the interaction effect on the infection of walnut shoots when inoculations were conducted by using conidial suspensions (Fig. 2B). Inoculation with $N$. mediterraneum only, the interaction of $N$. mediterraneum and D. rhusicola when both species were inoculated at the same time, and $N$. mediterraneum inoculated 4 days before $D$. rhusicola caused the longest $(P<0.0001)$ lesions ( $\approx 9 \mathrm{~cm}$ at 4 weeks after inoculation). Shoots inoculated with $D$. rhusicola showed the lowest disease severity value, with a necrosis length of $3.4 \mathrm{~cm}$, followed by the interaction treatment in which $D$. rhusicola was inoculated 4 days before $N$. mediterraneum, showing a necrosis length of $4.7 \mathrm{~cm} 4$ weeks after inoculation. In this last case, a delay in lesion development was also observed when inoculations were done by conidial suspension (Fig. 2B). Data on reisolations were obtained together from inoculated detached shoots by mycelial plugs and conidial suspension. Reisolation of $N$. mediterraneum was the most consistent (87.5 to 100\%), whereas the reisolation percentages of $D$. rhusicola were lower (21.5 to $87.5 \%$ ) than those observed for $N$. mediterraneum in all evaluated treatments. Under controlled conditions, $N$. mediterraneum and D. rhusicola were not reisolated from shoots inoculated with D. rhusicola or $N$.

Table 2. Effect of the interaction between Diaporthe rhusicola isolate $6 \mathrm{I} 31$ and Neofusicoccum mediterraneum isolate 5C87 on conidial germination 24 and $48 \mathrm{~h}$ after incubation

\begin{tabular}{llccc}
\hline & & \multicolumn{3}{c}{ Germination $(\%)^{\mathbf{z}}$} \\
\cline { 3 - 5 } Fungal species & Treatment & $\mathbf{2 4} \mathbf{~ h}$ & $\mathbf{4 8 ~ h}$ & Average \\
\hline D. rhusicola & Control & $72.3 \mathrm{a}$ & $97.8 \mathrm{a}$ & $85.1 \mathrm{a}$ \\
& Interaction & $18.3 \mathrm{~b}$ & $23.8 \mathrm{~b}$ & $21.0 \mathrm{~b}$ \\
\multirow{3}{*}{ N. mediterraneum } & Inhibition & 74.4 & 75.7 & 75.1 \\
& Control & $61.3 \mathrm{a}$ & $81.5 \mathrm{a}$ & $71.4 \mathrm{a}$ \\
& Interaction & $69.9 \mathrm{a}$ & $72.8 \mathrm{a}$ & $71.4 \mathrm{a}$ \\
& Inhibition & -14 & 11 & -1.5 \\
\hline
\end{tabular}

${ }^{\mathrm{z}}$ Values represent the means of two repetitions from three runs of each treatment. For each isolate, means in a column followed by the same lowercase letter do not differ significantly according to the chi-square test at $P=0.05$. mediterraneum alone, respectively, as well as from noninoculated control shoots (Table 3).

Inoculations under field conditions. Symptoms developed in shoots inoculated by conidial suspensions of both pathogens under field conditions were highly variable. For this reason, only shoots inoculated with mycelial plugs were evaluated for this experiment. A similar pattern was observed in the interaction effect on the infection of attached walnut shoots than that observed for detached shoots at 4 months after inoculation. Because there were no significant differences between the isolates of the same fungal species (KruskalWallis test, $P=0.0535$ and $P=0.0997$ for $N$. mediterraneum and $D$. rhusicola isolates, respectively), the treatments were grouped per fungal species. Inoculations with $N$. mediterraneum, and the interaction of $N$. mediterraneum and D. rhusicola when both species were inoculated at the same time, or when $N$. mediterraneum was inoculated at 4 days before $D$. rhusicola caused the longest lesions. For these treatments, the mean necrosis lengths observed on affected tissues were $2.3,2.6$, and $2.6 \mathrm{~cm}$, respectively, at 4 months after inoculation. Shoots inoculated with $D$. rhusicola showed the smallest lesion, with a necrosis length of $1.1 \mathrm{~cm}$, followed by the interaction treatment in which $D$. rhusicola was inoculated 4 days before $N$. mediterraneum, which showed a necrosis length of $2 \mathrm{~cm}$ at 4 months after inoculation. A delay in lesion development in shoots inoculated with $D$. rhusicola 4 days before $N$. mediterraneum was also observed under field conditions (Fig. 3). Reisolations showed that N. mediterraneum was the most prevalent species in field infections. When reisolations were done from the margin of the necrotic lesions, reisolation of $N$. mediterraneum ranged from 62.0 to $92.0 \%$, whereas D. rhusicola was reisolated with very low percentages (4.0 to $16.5 \%)$ only from two treatments. When reisolations were done from $2 \mathrm{~cm}$ beyond the margin of the necrotic lesions, reisolation of $N$. mediterraneum ranged from 33.3 to $71 \%$, whereas $D$. rhusicola was only reisolated with a very low percentage $(4.0 \%)$ only from two treatments. Finally, $N$. mediterraneum was also reisolated from the margin $(50 \%)$ and $2 \mathrm{~cm}$ beyond the margin $(33.3 \%)$ of necrotic lesions observed in noninoculated control shoots, probably owing to the natural contaminations that occurred in field conditions during the experimental period (Table 3).

Inoculation of walnut fruits. There were no significant differences between $D$. rhusicola isolates $(P=0.0901)$, but there were differences between $N$. mediterraneum isolates $(P=0.0011)$. Thus, treatments were analyzed separately for $N$. mediterraneum isolates $5 \mathrm{C} 87$ and 6I45. In all cases, the two fungal species and the interaction treatments tested produced a higher decayed area on the fruit surface than that observed in the noninoculated control fruit. For the treatments conducted with $N$. mediterraneum isolate $5 \mathrm{C} 87$, significant differences were observed among treatments tested $(P=0.0001)$. In this case, a similar pattern as that observed on inoculated walnut shoots was also observed in the evaluation of the interaction effect on inoculated hulls of walnut. In this tissue, inoculations with $N$. mediterraneum isolate $5 \mathrm{C} 87$ alone, and the interaction of $N$. mediterraneum isolate $5 \mathrm{C} 87$ and D. rhusicola when both species were inoculated at the same time, were the most severe treatments causing necrosis on the fruit surface, showing RAUDPC values of 87.3 and $76.8 \%$, respectively. Hulls of fruit inoculated with $D$. rhusicola showed the lowest RAUDPC (47.9\%), followed by the interaction treatment in which $D$. rhusicola was inoculated 4 days before $N$. mediterraneum isolate 5C87 (RAUCPC $=62.2 \%$ ). The delay in lesion development when $D$. rhusicola was inoculated 4 days before $N$. mediterraneum was also observed on inoculated immature hulls (Fig. 4A). However, when inoculations were done with $N$. mediterraneum isolate 6I45, only the interaction treatment between $N$. mediterraneum isolate $6 \mathrm{I} 45$ and $D$. rhusicola inoculating both species at the same time differed significantly $(P=0.0029)$ from the rest of the treatments, reaching the highest RAUDPC value (70.3\%). For the other treatments, the RAUDPC ranged from 49.9 to $55.0 \%$ for fruit inoculated with $D$. rhusicola and N. mediterraneum isolate 6I45, respectively (Fig. 4B).

Effect of age and tissue of the pruned shoots on pathogen infection. Because the interactions between the independent variables were significantly different $(P<0.05)$, treatment 
means of the internal and external lesion lengths were analyzed separately for each fungal species. For the $N$. mediterraneum infections, external necrosis only showed significant differences for the age of the shoots $(P<0.0001)$, with lesions on young shoots being longer than those observed on old shoots. However, internal necrosis showed significant differences for the age of the shoots $(P<$ $0.0001)$, for the inoculation site $(P=0.0307)$, and also for their interaction $(P=0.0473)$. In this case, internal lesions on young shoots were also longer than those observed on old shoots. Moreover, internal lesions that developed on young shoots that were inoculated on the pith tissue were significantly longer than those observed in young shoots inoculated on the bark. Nevertheless, no significant differences between internal necrosis length and inoculation site were observed on old shoots (Fig. 5A). Concerning infections with $D$. rhusicola, no significant differences were observed between the development of internal or external necrosis and the age of the shoots ( $P=0.0968$ and $P=0.1000$, respectively $)$, the inoculation site $(P=$ 0.4899 and $P=0.1437$, respectively), and their interactions $(P=$ 0.0533 and $P=0.0503$, respectively) (Fig. 5B). Finally, a positive linear correlation was observed between internal and external lesion length for $N$. mediterraneum ( $r=0.991 ; P=0.0087)$ as well as for $D$. rhusicola ( $r=0.967 ; P=0.0326)$ infections. $N$. mediterraneum was consistently reisolated (»65\%) from detached shoots inoculated with this fungal species, obtaining $100 \%$ of reisolation from 3- to 4-yearold branches inoculated in the pith. The percentage of reisolation of D. rhusicola from detached shoots inoculated with this fungal species was $20 \%$ for all treatment combinations.

\section{Discussion}

In this study, our main goal was to determine the effect of the interaction of $D$. rhusicola and $N$. mediterraneum on the development of branch dieback of English walnut in California. Several experiments were carried out under laboratory or natural field conditions to determine the effect of this interaction on the infection of shoots and hulls of walnut. Experiments in vitro to evaluate the optimum mycelial growth temperature and the interaction effect of both species on spore germination were also performed. In addition, the effect of age and type of tissue (bark surface or pith) of pruned shoots on $D$. and $N$. mediterraneum infection was also studied. To our knowledge, this is the first study evaluating the effect of the interaction of $D$. rhusicola and $N$ mediterraneum on the infection of branches of English walnut as well as on other woody species. Moreover, studies of the interaction between different wood pathogens and of the effect of pruning wounds on pathogen infections will contribute to improve this knowledge in other woody and nut crops.

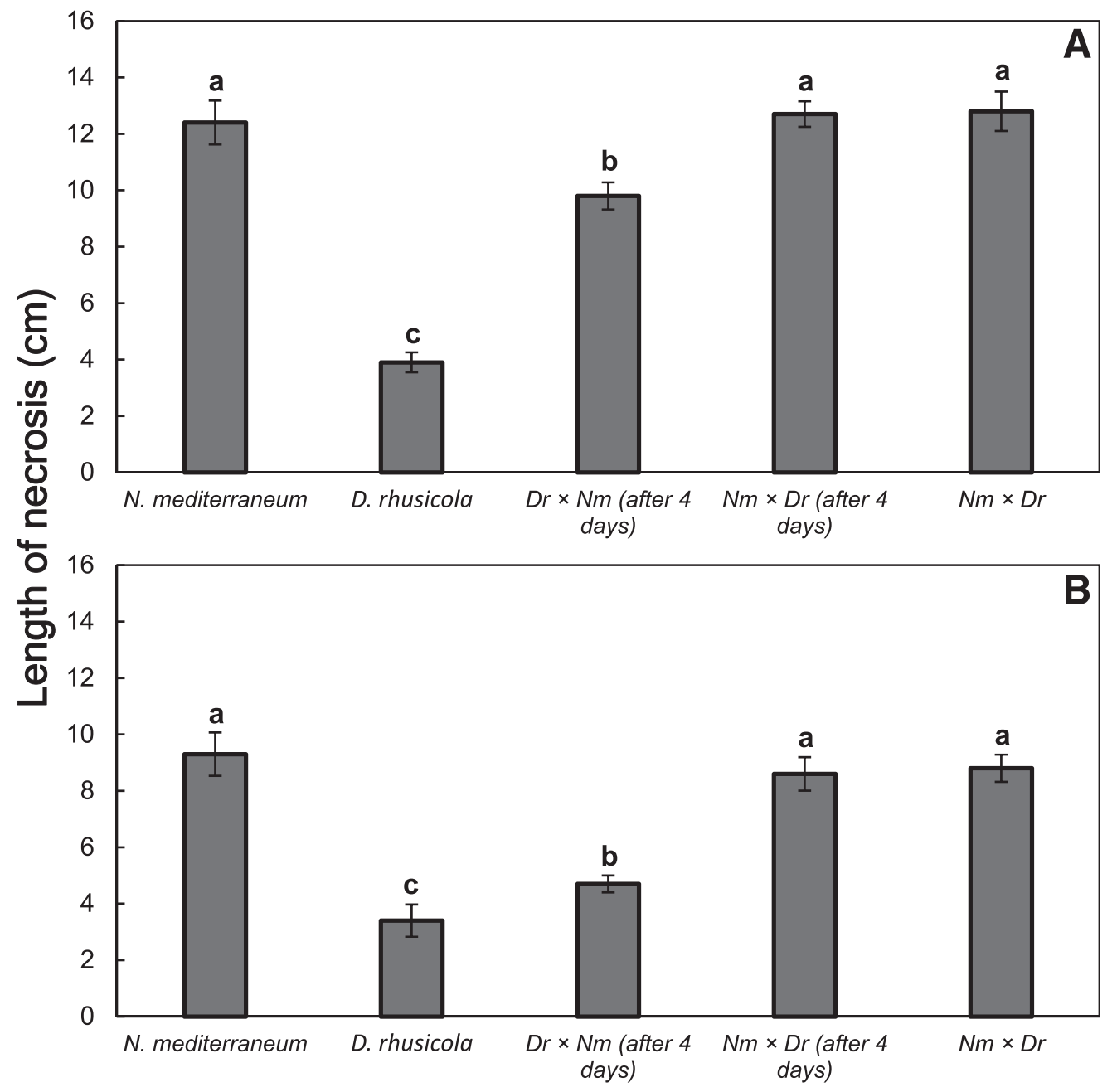

\section{Treatments}

Fig. 2. Disease severity on detached shoots of walnut cultivar Vina at 4 weeks after inoculation with mycelial plugs (A) or conidial suspensions (B) of Neofusicoccum mediterraneum and Diaporthe rhusicola. Single inoculations of N. mediterraneum and D. rhusicola and the following interaction treatments were conducted: D. rhusicola and N. mediterraneum, and $N$. mediterraneum and $D$. rhusicola, with the second species being inoculated at 4 days after the first one in each case; and $N$. mediterraneum and $D$. rhusicola both inoculated at the same time. Columns represent the mean values of 15 replicate shoots. Columns with common letters do not differ significantly according to Fisher's protected LSD test at $P=$ 0.05. Vertical lines on the columns are the standard error of the mean. 
First, the effect of temperature on mycelial growth of the four isolates selected for this study was evaluated in order to improve our knowledge about the biology of D. rhusicola and $N$. mediterraneum. The optimum temperature for mycelial growth of the isolates showed the diversity existing among them, since there was a significant difference between the two isolates of each fungal species. However, the average optimum temperature for the four isolates was $\sim 26^{\circ} \mathrm{C}$, with $30.4^{\circ} \mathrm{C}$ being obtained for $N$. mediterraneum isolate $6 \mathrm{I} 45$. Similar results were obtained by Moral et al. (2010), who evaluated the effect of temperature on mycelial growth of Botryosphaeriaceae fungi recovered from olive infected by branch dieback in California. These authors indicated that the optimum growth temperature for $N$. mediterraneum isolates collected from olive ranged from 27 to $29^{\circ} \mathrm{C}$.

These findings suggest that Botryosphaeriaceae fungi as well as some Diaporthaceae isolates are well adapted to the warm temperatures that occur in the olive and walnut growing areas in California during late spring and summer, when cankers start to develop in the infected shoots.

Table 3. Reisolation of Neofusicoccum mediterraneum and Diaporthe rhusicola from detached and attached shoots of English walnut inoculated to evaluate their interaction on walnut infection

\begin{tabular}{|c|c|c|c|c|}
\hline \multirow[b]{2}{*}{ Treatment $^{\mathrm{y}}$} & \multirow[b]{2}{*}{ Experiment } & \multirow[b]{2}{*}{ Isolation point } & \multicolumn{2}{|c|}{ Reisolation $(\%)^{\mathrm{z}}$} \\
\hline & & & N. mediterraneum & D. rhusicola \\
\hline \multirow[t]{3}{*}{ N. mediterraneum } & Detached shoots & Margin of necrotic lesions & 100 & 0.0 \\
\hline & Attached shoots & Margin of necrotic lesions & 75.0 & 0.0 \\
\hline & & $2 \mathrm{~cm}$ beyond the margin of necrotic lesions & 58.0 & 0.0 \\
\hline \multirow[t]{3}{*}{ D. rhusicola } & Detached shoots & Margin of necrotic lesions & 0.0 & 87.5 \\
\hline & Attached shoots & Margin of necrotic lesions & 50.0 & 16.5 \\
\hline & & $2 \mathrm{~cm}$ beyond the margin of necrotic lesions & 42.6 & 0.0 \\
\hline \multirow{3}{*}{$\begin{array}{l}\text { D. rhusicola } \times N \text {. mediterraneum (after } 4 \\
\text { days) }\end{array}$} & Detached shoots & Margin of necrotic lesions & 87.5 & 57.5 \\
\hline & Attached shoots & Margin of necrotic lesions & 79.0 & 4.0 \\
\hline & & $2 \mathrm{~cm}$ beyond the margin of necrotic lesions & 42.0 & 4.0 \\
\hline \multirow{3}{*}{$\begin{array}{l}\text { N. mediterraneum } \times \text { D. rhusicola } \text { (after } 4 \\
\text { days) }\end{array}$} & Detached shoots & Margin of necrotic lesions & 91.5 & 51.5 \\
\hline & Attached shoots & Margin of necrotic lesions & 62.0 & 0.0 \\
\hline & & $2 \mathrm{~cm}$ beyond the margin of necrotic lesions & 50.0 & 0.0 \\
\hline \multirow[t]{3}{*}{ N. mediterraneum $\times$ D. rhusicola } & Detached shoots & Margin of necrotic lesions & 87.0 & 55.0 \\
\hline & Attached shoots & Margin of necrotic lesions & 92.0 & 0.0 \\
\hline & & $2 \mathrm{~cm}$ beyond the margin of necrotic lesions & 71.0 & 4.0 \\
\hline \multirow{3}{*}{ Control } & Detached shoots & Margin of necrotic lesions & 0.0 & 0.0 \\
\hline & Attached shoots & Margin of necrotic lesions & 50.0 & 0.0 \\
\hline & & $2 \mathrm{~cm}$ beyond the margin of necrotic lesions & 33.3 & 0.0 \\
\hline
\end{tabular}

${ }^{\mathrm{y}} N$. mediterraneum $=$ single inoculation of $N$. mediterraneum $; D$. rhusicola $=$ single inoculation of $D$. rhusicola; $D$. rhusicola $\times N$. mediterraneum (after 4 days) and $N$. mediterraneum $\times D$. rhusicola (after 4 days) $=D$. rhusicola and $N$. mediterraneum and $N$. mediterraneum and $D$. rhusicola, respectively, with the second species being inoculated at 4 days after the first one in each case; . mediterraneum $\times D$. rhusicola $=$ both inoculated at the same time; and control $=$ shoots treated with potato dextrose agar plugs or sterile distilled water as the control.

${ }^{\mathrm{z}}$ For each treatment, experiment, and isolation point combination, the reisolation percentage was calculated as follows: Reisolation $(\%)=([$ number of positive inoculation points/numbers of inoculation points $] \times 100$ ).

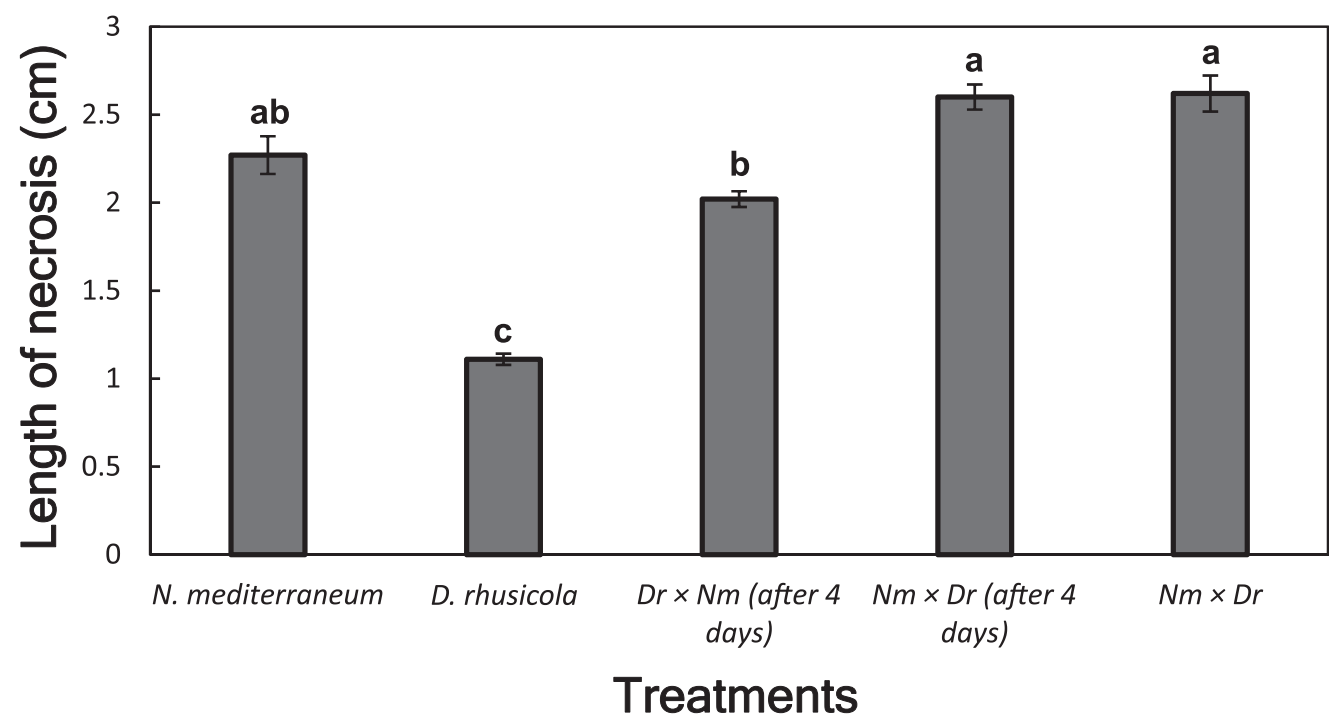

Fig. 3. Disease severity on attached shoots of walnut cultivar Vina 4 months after inoculation with mycelial plugs of Neofusicoccum mediterraneum and Diaporthe rhusicola. Single inoculations of $N$. mediterraneum and $D$. rhusicola and the following interaction treatments were conducted: $D$. rhusicola and $N$. mediterraneum, and $N$. mediterraneum and $D$. rhusicola, with the second species being inoculated at 4 days after the first one in each case; and N. mediterraneum and $D$. rhusicola both inoculated at the same time. Columns represent the mean values of 15 replicate shoots. Columns with common letters do not differ significantly according to Dunn's test at $P=0.05$. Vertical lines on the columns are the standard error of the mean. 
In the in vitro experiments, the spore germination of $D$. rhusicola was reduced by the presence of $N$. mediterraneum spores. Conversely, D. rhusicola spores had no effect on spore germination of $N$. mediterraneum. This information was fundamental to understand the following interaction experiments conducted in vivo. Evaluating the interaction effect on the infection of walnut shoots by $N$. mediterraneum and D. rhusicola showed consistent results in all of the experiments conducted. Overall, inoculations with $N$. mediterraneum, and the interaction between $N$. mediterraneum and D. rhusicola when both species were inoculated at the same time, or when N. mediterraneum was inoculated 4 days before $D$. rhusicola were the most virulent treatments affecting necrosis development. The high virulence of $N$. mediterraneum infecting English walnut shoots was also reported previously (Chen et al. 2014a; Trouillas et al. 2010). Single inoculation with $D$. rhusicola showed the lowest disease severity values. These results are in concordance with those obtained by Chen et al. (2014a), who demonstrated the pathogenicity of a broad range of Botryosphaeriaceae and Diaporthaceae isolates in English walnut. These authors demonstrated that $D$. rhusicola is weakly virulent to walnut (Chen et al. 2014a). Remarkably, the presence of both Botryosphaeriaceae and Diaporthaceae families is common in English walnut orchards in California (Chen et al. 2014a; Trouillas et al. 2010). In fact, in our study, the interaction treatment in which
D. ruschicola was inoculated 4 days before $N$. mediterraneum caused a larger lesion than that caused in shoots treated with single inoculations of D. rhusicola. However, in this interaction treatment, the lesion length was less than that observed in the other two interaction treatments evaluated. This lesser virulence on shoots for this interaction treatment could be related to the inhibition effect on the spore germination of $D$. rhusicola observed in the interaction treatments conducted in vitro. On the other hand, this result suggests that $D$. rhusicola can affect the colonization and pathogenicity of $N$. mediterraneum when infection and tissue colonization by $D$. rhusicola occur prior to $N$. mediterraneum. This could be attributable to the fact that the saprophytic capacity of $N$. mediterraneum colonizing dead tissues previously infected by $D$. rhusicola is higher than the pathogenic activity of $N$. mediterraneum. However, we cannot discard the possibility of an antagonistic effect of $D$. rhusicola against $N$. mediterraneum. In this way, in vitro studies conducted by Prada et al. (2009) evaluating the interaction between Diaporthe species and Phytophthora infestans showed that the endophytic fungi Diaporthe species have an antagonistic effect on mycelial growth development against the pathogen. Moreover, these same authors detected a gene from Diaporthe species encoding an amylase that was differentially expressed during the biotic interaction with $P$. infestans (Prada et al. 2009). This suggests that endophytic fungi such as
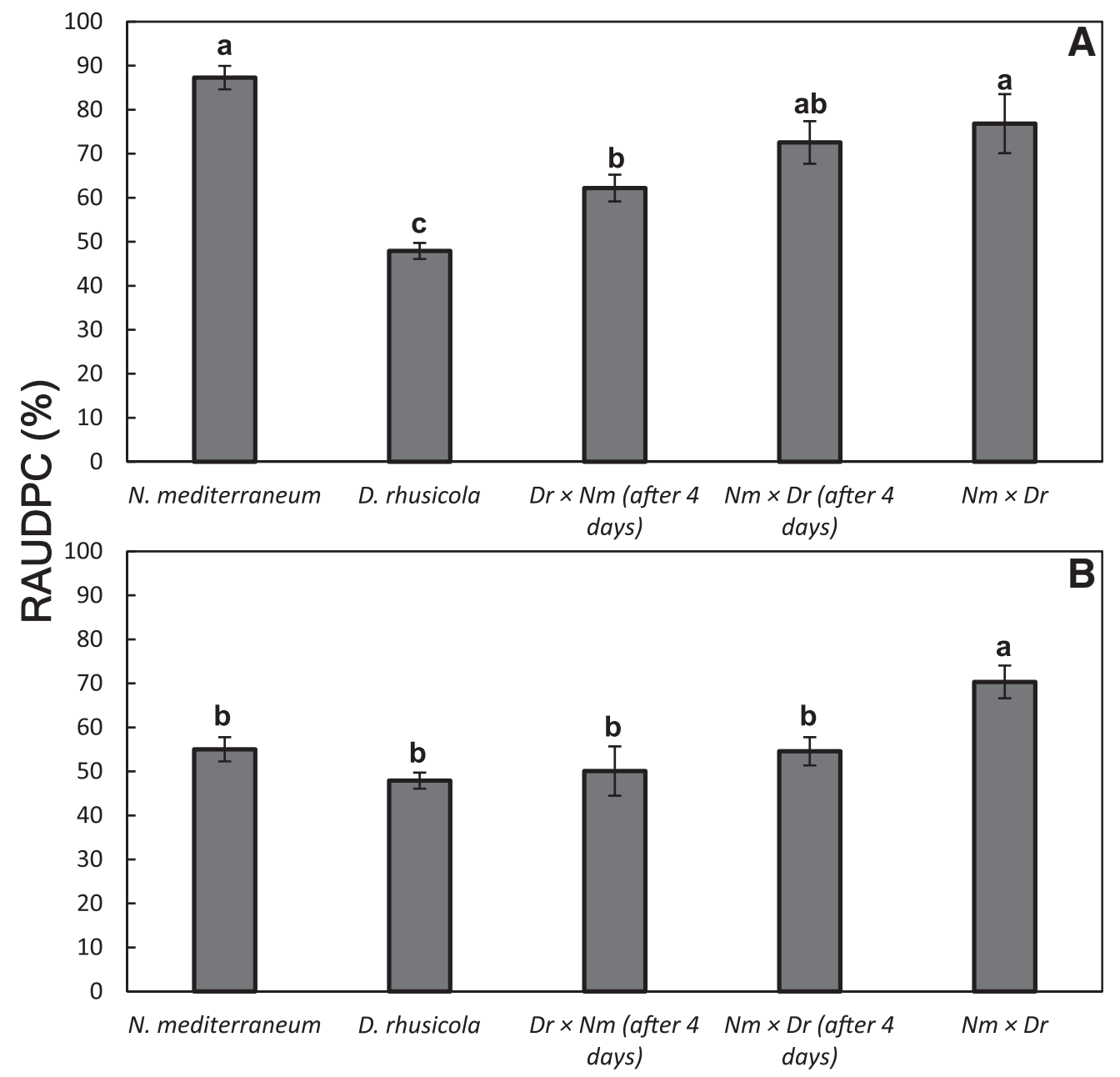

\section{Treatments}

Fig. 4. Relative area under the disease progress curve (RAUDPC) on hulls of the fruit of walnut cultivar Vina inoculated with conidial suspensions of two Diaporthe rhusicola isolates and Neofusicoccum mediterraneum isolates $5 \mathrm{C} 87(\mathrm{~A})$ and $6145(\mathrm{~B})$. Single inoculations of $D$. rhusicola and $N$. mediterraneum and the following interaction treatments were conducted: D. rhusicola and N. mediterraneum, and N. mediterraneum and D. rhusicola, with the second species being inoculated at 4 days after the first in each case; and $N$. mediterraneum and D. rhusicola both inoculated at the same time. Columns represent the mean values of 30 replicate fruits. Columns with common letters do not differ significantly according to Fisher's protected LSD test at $P=0.05$. Vertical lines on the columns are the standard error of the mean. 
Diaporthe species can produce a great variety of secondary metabolites causing antagonism against other fungi when a biotic interaction occurs. Thus, these previous results support the need to conduct further research elucidating the antagonistic effect of Diaporthe species against Botryosphaeriaceae in walnut infections and perhaps in other pathosystems.

The results of the interaction experiments on inoculated green hulls of walnut showed a similar pattern to those observed on inoculated shoots. Fruits inoculated with $N$. mediterraneum alone or combined with $D$. rhusicola in the interaction treatments showed the decay of the whole hull or a majority of the inoculated hulls in less than 3 weeks after inoculation with conidial suspensions, whereas fruit inoculated with $D$. rhusicola alone showed markedly lower hull rot. Our results are in agreement with those obtained by Chen et al. (2014a), who demonstrated that Botryosphaeriaceae species, including $N$. mediterraneum, can rot the whole hull of the fruit at 3 weeks after inoculation with mycelial plugs, whereas D. rhusicola isolates cannot (Chen et al. 2014a).
It is well known that species belonging Botryosphaeriaceae and Diaporthaceae develop and survive as saprophytic microorganisms on dead plant tissues, developing fruiting bodies that can produce viable spores (J. Moral et al., unpublished data). Subsequently, these spores are an important inoculum source to cause branch dieback on English walnut, with the pruning wounds being a potential infection court via which the spores can enter, infect, and colonize walnut shoots (Michailides and Hasey 2010). The effect of pruning wounds on the infection of fungal trunk pathogens such as Botryosphaeriaceae has already been well demonstrated in Prunus species (Olmo et al. 2017; T. J. Michailides, unpublished data) and grapevine (Agustí-Brisach et al. 2015; Rolshausen et al. 2010). However, to our knowledge, this study is the first to demonstrate the effect of pruning wounds as well as the influence of other factors such as the susceptibility of the wounded bark or pith to infection by $D$. rhusicola and N. mediterraneum on English walnut. The inoculations conducted with both of these species in the wounded bark or pith of walnut shoots of two age categories just after pruning showed
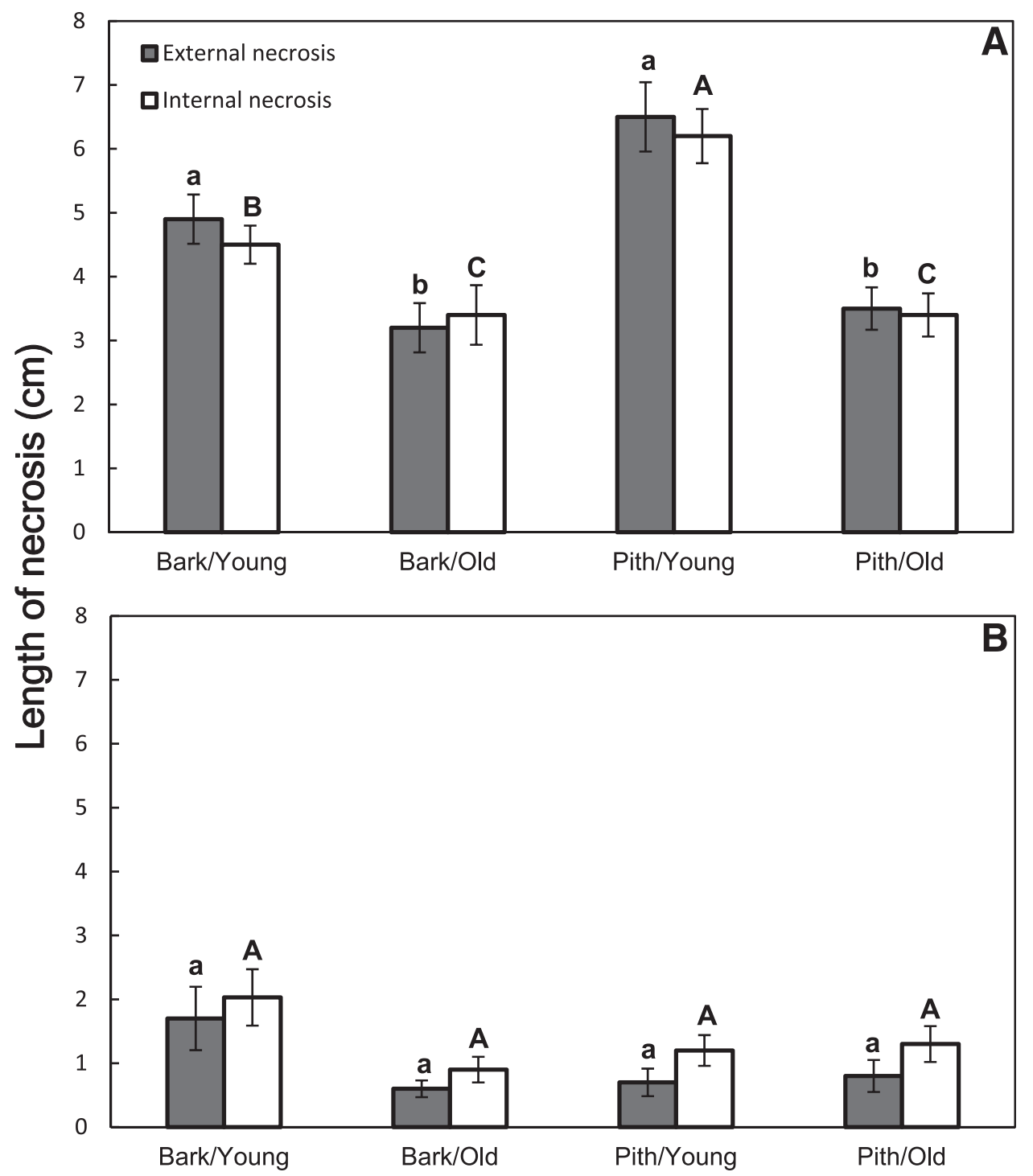

Tissue/Age of pruned shoots

Fig. 5. Pruning effect on disease severity (length of external and internal necrosis in centimeters) on detached shoots of walnut cultivar Chandler at 3 weeks after inoculation with conidial suspensions of Neofusicoccum mediterraneum isolate $5 \mathrm{C} 87$ (A) and Diaporthe rhusicola isolate $6 \mathrm{l31}$ (B). Inoculations were done just after pruning on the bark or on the pith of shoots of two age categories (young: 1 to 2 years old; and old: 3 to 4 years old). Columns represent the mean values of 15 replicate shoots. For each type of necrosis (external or internal), columns with common letters do not differ significantly according to Fisher's protected LSD test at $P=0.05$. Vertical lines on the columns are the standard error of the mean. 
different results for each one. Inoculations conducted with $D$, rhusicola did not show any significant differences between the development of internal or external necrosis and the age of the shoots, or the susceptibility of bark and pith on the infection. However, for $N$. mediterraneum, 1- to 2-year-old shoots were more susceptible to infection and colonization by the fungus than 3- to 4-year-old shoots for both external and internal necrosis development. Moreover, differences in susceptibility to infection between the inoculation site (bark or pith) were also observed for internal necrosis development on young shoots inoculated with $N$. mediterraneum. In fact, it is a frequent phenomenon in commercial orchards to find the infections advancing through the pith more so than the woody tissues of the shoots (Fig. 1).

The information obtained in this work is relevant and demonstrates the interaction between $D$. rhusicola and $N$. mediterraneum on the infection of shoots and hulls of English walnut. Our results suggest that special attention should be given to the possible retarding or antagonistic effect of $D$. rhusicola against $N$. mediterraneum observed when the infection of $D$. rhusicola occurs before that of $N$. mediterraneum. However, further research is needed to elucidate the cause of this interaction effect between both species in the infection of walnut shoots. Moreover, the studies conducted to determine the effect of pruning wounds suggest the need to protect wounds just after pruning in order to avoid infection by Botryosphaeriaceae fungi mainly. In addition, when pruning an infected shoot, one has to cut below the obvious external margin of the canker, since the infection in the pith is frequently beyond the infection of the wood (margin of canker). In general, experience shows that pruning 5 to $7 \mathrm{~cm}$ below the external margin of the cankers is effective in removing the infection entirely (T. J. Michailides, unpublished data). Altogether, this information is essential to better understand the complex situation of this important disease in walnut orchards in California and is helpful in making appropriate decisions for the development of management strategies to control branch dieback.

\section{Acknowledgments}

The authors thank L. Doster for her skillful technical assistance. This study was conducted at the Kearney Agricultural Research and Extension Center at University of California, Davis, where C. Agustí-Brisach served as a visiting scientist from March to July 2017

\section{Literature Cited}

Agustí-Brisach, C., León, M., García-Jiménez, J., and Armengol, J. 2015. Detection of grapevine fungal trunk pathogens on pruning shears and evaluation of their potential for spread of infection. Plant Dis. 99:976-981.

Analytical Software. 2013. Statistix 10 User's Manual. Analytical Software, Tallahassee, FL.

California Department of Food and Agriculture. 2017. Page 11 in: California Agricultural Statistics Review 2016-2017. California Department of Food and Agriculture, Sacramento, CA.

Campbell, C. L., and Madden, L. V. 1990. Introduction to Plant Disease Epidemiology. John Willey \& Sons, New York.

Chen, S. F., Morgan, D. P., Hasey, J. K., Anderson, K., and Michailides, T. J. 2014a. Phylogeny, morphology, distribution, and pathogenicity of Botryosphaeriaceae and Diaporthaceae from English walnut in California. Plant Dis. 98:636-652.
Chen, S. F., Morgan, D. P., and Michailides, T. J. 2014b. Botryosphaeriaceae and Diaporthaceae associated with panicle and shoot blight of pistachio in California, USA. Fungal Divers. 67:157-179.

Gramaje, D., Agustí-Brisach, C., Pérez-Sierra, A., Moralejo, E., Olmo, D., Mostert, L., Damm, U., and Armengol, J. 2012. Fungal trunk pathogens associated with wood decay of almond trees on Mallorca (Spain). Persoonia 28:1-13.

Hau, B., and Kranz, J. 1990. Mathematics and statistics for analyses in epidemiology. Pages 12-52 in: Epidemics of Plant Diseases. J. Kranz, ed. Springer-Verlag, Berlin.

Inderbitzin, P., Bostock, R. M., Trouillas, F. P., and Michailides, T. J. 2010. A sixlocus phylogeny reveals high species diversity in Botryosphaeriaceae from California almond. Mycologia 102:1350-1368.

Li, G. Q., Liu, F. F., Li, J. Q., Liu, Q. L., and Chen, S. F. 2015. Characterization of Botryosphaeria dothidea and Lasiodiplodia pseudotheobromae from English walnut in China. J. Phytopathol. 164:348-353.

López-Moral, A., Raya-Ortega, M. C., Agustí-Brisach, C., Roca, L. F., Lovera, M., Luque, F., Arquero, O., and Trapero, A. 2017. Morphological, pathogenic, and molecular characterization of Colletotrichum acutatum isolates causing almond anthracnose in Spain. Plant Dis. 101:2034-2045.

Ma, Z., and Michailides, T. J. 2002. Characterization of Botryosphaeria dothidea isolates collected from pistachio and other plant hosts in California Phytopathology 92:519-526.

McDonald, V., and Eskalen, A. 2011. Botryosphaeriaceae species associated with avocado branch cankers in California. Plant Dis. 95:1465-1473.

Michailides, T. J., and Hasey, J. 2010. Botryosphaeria and Phomopsis cankers of walnuts in California. Walnut husk fly field meeting. University of California Cooperative Extension, Colusa, CA.

Moral, J., Muñoz-Díez, C., González, N., Trapero, A., and Michailides, T. J. 2010 Characterization and pathogenicity of Botryosphaeriaceae species collected from olive and other hosts in Spain and California. Phytopathology 100: 1340-1351.

Olmo, D., Gramaje, D., and Armengol, J. 2017. Evaluation of fungicides to protect pruning wounds from Botryosphaeriaceae species infections on almond trees. Phytopathol. Mediterr. 56:77-86.

Olson, W. H., and Buchner, R. P. 2002. Leading edge of plant protection for walnuts. HortTechnology 12:615-618.

Prada, H., Ávila, L., Sierra, R., Bernal, A., and Restrepo, S. 2009. Caracterización morfológica y molecular del antagonismo entre el endofito Diaporthe sp. aislado de frailejón (Espeletia sp.) y el fitopatógeno Phytophthora infestans. Rev. Iberoam. Micol. 26:198-201.

Rolshausen, P. E., Úrbez-Torres, J. R., Rooney-Latham, S., Eskalen, A., Smith, R. J., and Gubler, W. D. 2010. Evaluation of pruning wound susceptibility and protection against fungi associated with grapevine trunk diseases. Am. J. Enol. Vitic. 61:113-119.

Slippers, B., and Wingfield, M. J. 2007. Botryosphaeriaceae as endophytes and latent pathogens of woody plants: Diversity, ecology and impact. Fungal Biol. Rev. 21:90-106.

Steel, R. G. D., and Torrie, J. H. 1985. Bioestadística, 2nd ed. McGraw-Hill, Bogotá, Colombia.

Teviotdale, B. L., Michailides, T. J., and Pscheidt, J. W. 2002. Compendium of Nut Crop Diseases in Temperate Zones. American Phytopathological Society Press, St. Paul, MN.

Trouillas, F. P., Úrbez-Torres, J. R., Peduto, F., and Gubler, W. D. 2010. First report of twig and branch dieback of English walnut (Juglans regia) caused by Neofusicoccum mediterraneum in California. Plant Dis. 94:1267.

Úrbez-Torres, J. R., and Gubler, W. D. 2009. Pathogenicity of Botryosphaeriaceae species isolated from grapevine cankers in California. Plant Dis. 93:584-592.

Úrbez-Torres, J. R., Leavitt, G. M., Voegel, T. M., and Gubler, W. D. 2006. Identification and distribution of Botryosphaeria spp. associated with grapevine cankers in California. Plant Dis. 90:1490-1503.

Úrbez-Torres, J. R., Peduto, F., Vossen, P. M., Krueger, W. H., and Gubler, W. D. 2013. Olive twig and branch dieback: Etiology, incidence, and distribution in California. Plant Dis. 97:231-244. 